\title{
Diagnostic Test of PIVKA-II as A Tumor Marker for Hepatocellular Carcinoma
}

\author{
I Putu Adi Santosa ${ }^{1}$, Dwi Priyadi Djatmiko ${ }^{1}$, Elvin Richela Lawanto ${ }^{1}$, Bogi Pratomo ${ }^{2}$, Hani Susianti ${ }^{1}$ \\ ${ }^{1}$ Department of Clinical Pathology Laboratory, Faculty of Medicine, Brawijaya University/Dr. Saiful Anwar General Hospital Malang, East \\ Java, Indonesia. E-mail: hanisusianti@yahoo.com \\ ${ }^{2}$ Division of Gastroenterohepatology, Department of Internal Medicine, Faculty of Medicine, Brawijaya University/Dr. Saiful Anwar General \\ Hospital Malang, East Java, Indonesia
}

\begin{abstract}
Alpha-Fetoprotein (AFP) is a tumor marker that has been widely used for HCC, but there has been no increased AFP in $35-45 \%$ patients with HCC. Protein induced by vitamin K absence or antagonist II (PIVKA-II) is abnormal prothrombin secreted in $\mathrm{HCC}$ and is expected to be used as a diagnostic marker of HCC. The objective of this study was to compare serum PIVKA-II levels in the patients with HCC, cirrhosis, and healthy control and determine the diagnostic value of PIVKA-II for hepatocellular carcinoma. This was a cross-sectional, analytical observational study to identify the diagnostic value of PIVKA-II for HCC diagnosis. The diagnosis of 20 cirrhotic patients and 15 patients with HCC was established by using medical history, physical examination, and additional tests according to the diagnosis criteria. A group of 12 individuals with normal liver function was used as healthy control subjects. Serum PIVKA-II levels were analyzed with the immunoassay method. For the comparison study, the independent-samples Kruskal Wallis test was used. Also, to determine sensitivity, specificity, positive and negative predictive value (PPV and NPV), ROC curve analysis, and $2 \times 2$ contingency table was used. The serum PIVKA-II levels in the patients with HCC were significantly higher than in cirrhotic patients $(p=0.000)$ and healthy control $(p=0.000)$. Sensitivity, specificity, PPV, and NPV of PIVKA-II for diagnosis of HCC in cirrhotic patients at a cut-off value of $140.85 \mathrm{mAU} / \mathrm{mL}$ were $93.33 \%, 75 \%, 73.68 \%$, and $93.75 \%$, respectively (AUC=0.87).PIVKA-II had a high diagnostic value for HCC diagnosis. Diagnostic tests that compare serum PIVKA-II levels in any size of HCC nodules might be needed in the future.
\end{abstract}

Keywords: PIVKA-II, AFP, hepatocellular carcinoma, diagnosis

\section{INTRODUCTION}

Hepatocellular Carcinoma (HCC) is the fifth most frequent malignancy in male and seventh in females. As the third most frequent malignancy in the world, it becomes the leading cause of death. There is an increasing incidence of HCC in East and Southeast Asia, as well as European countries with medium and low Gross Domestic Product (GDP). ${ }^{1}$

Hepatocellular carcinoma patients continued to increase from 2010 to 2013 in Indonesia, specifically in the Dharmais Cancer Hospital. ${ }^{2}$ The magnitude of the problem will undoubtedly have a profound impact on public health issues, productivity, life expectancy, and other socioeconomic aspects. ${ }^{3}$ In general, patients with HCC frequently come late to the doctor, and early detection of this disease remains a problem to date.

Alpha-Fetoprotein (AFP) is widely used as a tumor marker for diagnosing $\mathrm{HCC}$, but 35 to 45 percent of patients with HCC show no increased AFP levels. This fact leads to the limited use of the clinical value of AFP. ${ }^{4}$

Des-gamma-carboxyprothrombin (DCP), also known as a protein induced by vitamin $\mathrm{K}$ absence or antagonist II (PIVKA-II), is an abnormal form of prothrombin. In normal liver, prothrombin precursors undergo post-translated carboxylation (addition of carboxylic acid group) by gamma-glutamyl carboxylase before being released into peripheral blood. Carboxylase-dependent vitamin $\mathrm{K}$ which is responsible for carboxylation is not found in HCC, leading to the secretion of abnormal prothrombin. ${ }^{5}$

The meta-analysis study by Zhu et al. suggested that PIVKA-II had sufficient diagnostic value; therefore, measurement of PIVKA-II levels can be used as an alternative test to diagnose $\mathrm{HCC} .{ }^{5}$ To date, only few studies were performed to determine the diagnosis value of PIVKA-II as a tumor marker of HCC in Indonesia.

This research aimed to compare serum PIVKA-II 
levels in the patients with HCC, cirrhosis, and healthy control to determine the diagnostic value of PIVKA-II for HCC in cirrhotic patients.

\section{METHODS}

The study protocol was approved by the ethical committee of health research of Dr. Saiful Anwar General Hospital, Malang, East Java, Indonesia with number No. 400/195/K.3/302/2017. Informed consent was obtained from all patients who participated in the study. This research was performed at the Central Laboratory Installation of Dr. Saiful Anwar General Hospital, Malang, East Java, Indonesia, from July to December 2016. There were $15 \mathrm{HCC}$ patients and 20 cirrhotic patients within six months. This research used a cross-sectional observational analytic design with consecutive sampling. The subjects who met the inclusion and exclusion criteria were examined the serum PIVKA-II levels with TOSOH AIA-Pack by immunoassay method. Inclusion criteria were cirrhotic patients and/or HCC who underwent treatment in Dr. Saiful Anwar General Hospital, Malang, East Java, Indonesia. Exclusion criteria were patients suffering from malnutrition and HIV/AIDS. The diagnosis of cirrhosis and $\mathrm{HCC}$ was based on anamnesis, physical, laboratory, and radiology examinations. The subjects were divided into two groups, cirrhosis group consisting of 20 patients and HCC group composed of 15 patients. A group of 12 individuals with normal liver function was used as healthy control subjects. Serum samples of patients were stored at $-80^{\circ} \mathrm{C}$ until assayed. After the results of serum PIVKA-II levels were obtained, data were analyzed using SPSS 20 software.

Shapiro-Wilk (data<50) was employed for the data normality test, and univariate analysis was then used to determine the frequency distribution of the dependent variable, the median of serum PIVKA-II

Table 1. Subjects characteristics levels. Normally distributed data were further analyzed with a oneway ANOVA test using computer software. Non-parametric test, Kruskal Wallis test, was used due to abnormal distribution of data. Furthermore, the ROC curve analysis was performed on existing serum PIVKA-II level data to determine optimal cut-off to diagnose HCC in cirrhotic patients.

\section{RESULTS AND DISCUSSION}

There were total of 47 subjects involved in this study, consisting of fifteen (31.92\%) patients diagnosed with HCC and 20 (42.55\%) patients with cirrhosis. Besides, a group of 12 (25.53\%) individuals with normal liver function were used as healthy controls. No significant difference in ages was found among patients with HCC and cirrhosis. Table 1 summarizes the subject characteristics of each parameter analyzed.

The normality data test using the Shapiro-Wilk showed an abnormal serum PIVKA-II distribution $(p=0.000)$ among the three groups. There was a significant difference between PIVKA-II serum levels in patients with HCC, cirrhosis, and control $(p=0.000)$ based on the independent-samples Kruskal Wallis test. The ROC curve analysis in this study showed a potential ability of serum PIVKA-II levels to diagnose hepatocellular carcinoma in cirrhotic patients with an AUC value of 0.87 (Figure 1 ). With a cut-off level of $140.85 \mathrm{mAU} / \mathrm{mL}$, the serum PIVKA-II levels were able to diagnose hepatocellular carcinoma in cirrhotic patients with sensitivity, specificity, PPV, and NPV of 93.33\%; 75\%; 73.68\%; and $93.75 \%$, respectively.

The incidence of HCC is relatively high in Southeast Asia. There has been high mortality and low expectancy rate due to HCC, caused by the relationship between this malignancy with hepatitis $B$ and $C$ virus infections. ${ }^{6}$ Worldwide, $\mathrm{HCC}$ can be the most frequent case of tumor with a ratio of incidence between males and females of 4:1. Similarly, the

\begin{tabular}{lcccc}
\hline & HCC $(\mathbf{n}=\mathbf{1 5})$ & Cirrhosis $(\mathbf{n = 2 0})$ & $\begin{array}{c}\text { Healthy Controls } \\
(\mathbf{n = 1 2})\end{array}$ & P \\
\hline Gender & $13 ; 86.67 \%$ & $14 ; 70 \%$ & $5 ; 41.67 \%$ & \\
$\quad$ Male $(\mathrm{n} ; \%)$ & $2 ; 13.33 \%$ & $6 ; 30 \%$ & $7 ; 58.33 \%$ & \\
$\quad$ Female (n; \%) & $56.67 \pm 12.06$ & $55.45 \pm 10.48$ & $34.17 \pm 15.56$ & 0.86 \\
Age (mean \pm SD)(year) & $231,432.95 \pm 431,506.38$ & $78,813.74 \pm 303,316.88$ & $25.98 \pm 10.18$ & 0.00 \\
PIVKA-II (mean & & & & \\
(range)) (mAU/mL) & & $33.05(5-1,353,369)$ & $24.65(5-41.5)$ \\
PIVKA-II (median & $46,739.8(118.8-1,671,100.40)$ & & & \\
$($ range)) $(\mathrm{mAU} / \mathrm{mL})$ & & &
\end{tabular}




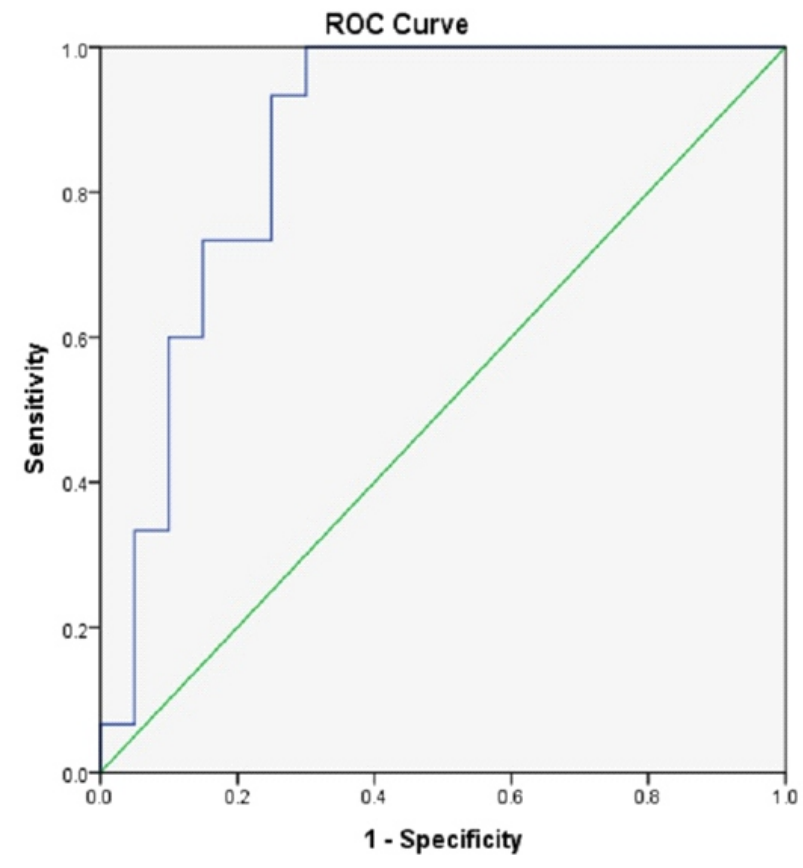

Figure 1. ROC curve of PIVKA-II for HCC in cirrhotic patients $(A \cup C=0.87)$

higher incidence in males was also found in this study. Hepatocellular carcinoma is dependent on sex hormones. However, the molecular mechanisms by which estrogens prevent and androgens promote liver cancer remain unclear.

Hepatocellular carcinoma generally develops in liver cirrhosis patients during the fifth or sixth decade of life. ${ }^{8}$ In more than $70 \%$ of cases, HCC develops from advanced liver cirrhosis. Patients with liver cirrhosis have a $30 \%$ increased risk of HCC within five years. This study described the diagnostic value of PIVKA-II for HCC in cirrhotic patients. The serum PIVKA-II level demonstrated relatively high sensitivity and specificity for diagnosing HCC in patients with cirrhosis. Furthermore, this study found that $\mathrm{HCC}$ was most commonly caused by hepatitis B virus infection (54\%) and hepatitis C (31\%). Hepatocellular carcinoma is rarely associated with chronic exposure to toxins or hereditary liver disease. Non-alcoholic fatty liver disease is a risk factor for the current prevalence of hepatocellular carcinoma. ${ }^{9}$ Des-gamma-carboxyprothrombin or protein induced by vitamin $\mathrm{K}$ absence, or antagonist II (PIVKA-II) is a form of immature prothrombin. PIVKA-II is nonfunctional prothrombin produced due to a deficiency of carboxylation of 10 glutamate acid residues in the $\mathrm{N}$-terminal domain of the molecule. In a normal liver, the prothrombin undergoes post-translational carboxylation before it is released into the peripheral blood. The carboxylation converts the glutamic acid residue by the specific amino-terminal to gamma-carboxy glutamic acid. Carboxylase is responsible for carboxylation and depends on vitamin $\mathrm{K}$. It is not found in hepatocellular carcinoma, leading to the secretion of abnormal prothrombin. Not only due to deficiency of vitamin $\mathrm{K}$, but the synthesis of excessive prothrombin precursors by HCC tissue of the patient also affects the production of PIVKA-II. Therefore, PIVKA-II is used as a biomarker of hepatocellular carcinoma. ${ }^{10}$

PIVKA-II is more sensitive (sensitivity of $100 \%$ with a cut-off value of $>53.7 \mathrm{ng} / \mathrm{mL}$ ) than AFP (sensitivity of $44 \%$, with a cut-off value of $>28 \mathrm{ng} / \mathrm{mL}$ ) for early detection of patients with hepatocellular carcinoma due to synthesis of excessive prothrombin precursors by tissue hepatocellular carcinoma of the patient which will certainly affect the production of PIVKA-II. ${ }^{11}$ In this study, serum PIVKA-II levels in hepatocellular carcinoma patients were significantly higher than that in cirrhotic patients and healthy controls. With a sensitivity of $93.33 \%$ and a cut-off level of $140.85 \mathrm{mAU} / \mathrm{mL}$, serum PIVKA-II levels were able to diagnose hepatocellular carcinoma in cirrhotic patients. In general, hepatocellular carcinoma patients come late to the doctor in Indonesia, leading to an increased cut-off level of serum PIVKA-II in this research.

PIVKA-II was able to differentiate hepatocellular carcinoma which was assessed histopathologically and had good diagnostic validity to evaluate portal vein thrombosis and to distinguish between early or advanced hepatocellular carcinoma. PIVKA-II levels were significantly associated with the size of a solitary tumor, which was increased 25 times higher in tumors with a size greater than two $\mathrm{cm}$ compared with tumors with a size less than two $\mathrm{cm}$. In addition, PIVKA-II can be used for screening in high-risk populations and diagnosis of early-stage disease to support curative therapy. ${ }^{11}$

PIVKA-II can be used as an early diagnosis of hepatocellular carcinoma in Indonesia. However, less specific PIVKA-II diagnostic values may be due to increased PIVKA-II levels in patients with vitamin $\mathrm{K}$ deficiency.

This study had several limitations that need further considerations. This study was merely a single-center investigation of a small sample, which limited the inferential analysis. The presented data might not be sufficient to conclude that serum PIVKA-II level should be used routinely for making a clinical decisions. Further research should be focused 
on a large population with a prospective cohort study to clarify the diagnostic and prognostic capabilities of individual and multiple biomarkers.

\section{CONCLUSION AND SUGGESTIONS}

Serum PIVKA-II seemed to be a promising diagnostic marker in cirrhotic patients with hepatocellular carcinoma. Further research was required to correlate between serum PIVKA-II level and the size of hepatocellular carcinoma nodule.

\section{ACKNOWLEDGMENT}

The authors acknowledge the lab technicians for technical assistance, and all physicians collaborating in the study.

\section{REFERENCES}

1. Bosetti C, Turati F, La Vecchia C. Hepatocellular carcinoma epidemiology. Best Practice \& Research Clinical Gastroenterology 2014; 28(5):753-70.

2. Health Ministry of the Republic of Indonesia. Stop Cancer. Jakarta, Data and Information Center, 2015; 1-6.

3. Health Ministry of the Republic of Indonesia. Hepatitis situation and analysis. Jakarta, Data and Information Center, 2014; 1-6.
4. Zhu R, Yang J, Xu L, Dai W, Wang F, Shen $M$, et al. Diagnostic performance of des- $\gamma$-carboxyprothrombin for hepatocellular carcinoma: A meta-analysis. Gastroenterology Research and Practice, 2014; 1-9.

5. Zhang YS, Chu JH, Cui SX, Song ZY, Qu XJ. Des-Y-carboxyprothrombin (DCP) as a potential autologous growth factor for the development of hepatocellular carcinoma. Cellular Physiology and Biochemistry, 2014; 34(3): 903-15.

6. Norsa'adah, Bachok, Nurhazalini-Zayani, Che Ghazali Che. Epidemiology and survival of hepatocellular carcinoma in North-East Peninsular Malaysia. Asian Pacific Journal of Cancer Prevention, 2013; 14(11): 6955-59.

7. Keng VW, Largaespada DA, Villanueva A. Why men are at higher risk for hepatocellular carcinoma?. Journal of Hepatology, 2012; 57(2): 453-4.

8. Kasper DL, Hauser SL, Jameson JL, Fauci AS, Longo DL, Loscalzo J. Harrison's principles of internal medicine. $19^{\text {th }}$ Ed., New York, McGraw-Hill Education, 2015; 2058.

9. Kirstein, MM. Vogel, A. The pathogenesis of hepatocellular carcinoma. Digestive Diseases 2014; 32(5): 545-553.

10. Murata K, Suzuki H, Okano H, Oyamada T, Yasuda Y, Sakamoto A. Hypoxia-induced des-y-carboxyprothrombin production in hepatocellular carcinoma. International Journal of Oncology, 2010; 36(1): 161-170.

11. Zakhary NI, Khodeer SM, Shafik HE, Malak CAA. Impact of PIVKA-II in diagnosis of hepatocellular carcinoma. Journal of Advanced Research, 2013; 4: 539-546. 